\footnotetext{
${ }^{*}$ Corresponding author
}

Abstract Stability, Virtual Inertia.

\title{
Frequency Support using Doubly Fed Induction and Reluctance Wind Turbine Generators
}

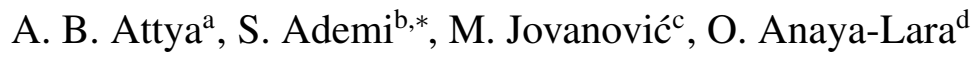 \\ ${ }^{a}$ Department of Electronic and Electrical Eng., University of Huddersfield, Huddersfield, UK \\ ${ }^{b}$ Warwick Manufacturing Group, University of Warwick, Coventry, UK \\ ${ }^{c}$ Faculty of Engineering and Environment, Northumbria University, Newcastle, UK \\ ${ }^{d}$ Department of Electronic and Electrical Engineering, University of Strathclyde, Glasgow, U.K.
}

This paper presents the comparative computer simulations of a commercial doublyfed induction generator (DFIG) and an emerging brushless doubly-fed reluctance generator (BDFRG) for grid-connected wind turbines in terms of frequency support based on the inertia emulation and blade pitching de-loading. The BDFRG features the low operation and maintenance cost by using a fractional inverter, and offers the high reliability of brushless structure with a simpler, more compact 2stage gearbox design whilst still ensuring competitive performance to its popular slip-ring companion. The implemented benchmark is carefully designed to ascertain the relative capabilities of the two wind turbine generator technologies in providing this ancillary service. The results reveal that in spite of the fundamentally different operating principles, the DFIG and the BDFRG are highly aligned from the viewpoint of power system applications.

Keywords: Wind Power, Doubly-Fed Machines, Ancillary Services, Frequency 
24

$25 D_{F}$ De-loading ratio

26 $H_{d}$ On-line inertia constant

$2 f_{d r o p \max }$ Full support frequency threshold

$28 f_{\text {low }}$ Frequency safe margin

$29 \quad f_{o}$ Nominal frequency

30 $\quad P_{c}$ Actual conventional generation in AC area

${ }_{31} P_{c}^{o}$ Installed conventional generation in AC area

32 $P_{W F}$ Wind farm generation

3з $P_{\text {ref }}^{o}$ Active power reference

${ }_{34} T_{e, m}$ Wind turbine electrical, mechanical torque

$35 T_{\text {gen }}$ Conventional generation torque

${ }_{36} T_{\text {ref }}^{o}$ Nominal reference torque

${ }_{37} D_{g}, D_{l}$ Dynamic load model parameters

$38 \quad J$ Moment of inertia

39 $\quad K_{\text {ex }}$ Extraction factor

40 $\quad R$ Droop of aggregate generator

${ }_{41} \Delta P$ Power mismatch

${ }_{42}$ BDFG Brushless Doubly-Fed Generator

4BDFIG Brushless Doubly-Fed Induction Generator

BDFRG Brushless Doubly-Fed Reluctance Generator

45 DFIG Doubly-Fed Induction Generator

${ }_{46}$ DFMs Doubly-Fed Machines 
47 KE Kinetic Energy

48 MPT Maximum Power Tracking

49 SGs Synchronous Generators

${ }_{50} \mathrm{RoCoF}$ Rate of Change of Frequency

51 TSOs Transmission System Operators

52 WFs Wind Farms

53 WS Wind Speed

${ }_{54}$ WTGs Wind Turbine Generators

\section{Introduction}

The foreseen proliferation of distributed generation, and the accompanying disconnection of conventional power plants, might seriously threaten the power system voltage and frequency stability $[1,2]$. Consequently, TSOs have been updating the grid codes to incorporate the new demands from classical SGs and WFs, while the manufacturers of WTGs are competing to assure the compliance of their products with the latest grid integration regulations, including the ability to participate in the frequency regulation [3]. The reduced global inertia is a critical challenge for the system stability to face with the increasing penetration of WFs in large power networks or island grids [4] [5]. To this extent, several control methods have been proposed with an incentive to allow the WTG to successfully tackle the frequency decay by imitating the inertia and primary responses of SGs in power stations $[3,4]$. Clear and well defined technical and legal rules and protocols are essential to avoid possible conflicts and malfunctions when such support 
methods are applied to large scale to ensure a smooth coordination between power systems and WFs during and shortly after frequency events [6].

This can be accomplished by maintaining a certain power reserve, or by releasing a portion of the stored KE in the WTG rotational parts [7]. The standard approaches taken are turbine blades pitching (e.g. de-loaded operation), emulated KE extraction (i.e. synthetic inertia), and tip-speed control [8]. Alternative innovative strategies have also been devised and studied recently [9]. The effectiveness of all these procedures is contingent upon the WTG responses to sudden changes in WS and/or torque (power) reference stipulated by the controller to produce the aimed power surge and help curtail the incurred frequency fluctuations in the best possible manner. Quantitative metrics of frequency support capabilities of various WTGs and WFs using different methodologies have been put forward in [10].

Some recent work touched upon the impact of the controllers gains to provide pitch de-loading and virtual inertia. The study derived the root-loci and produced time simulations of a wide range of the involved controllers parameters (e.g. pitching system, torque control and frequency support) to investigate whether the control system stability was aligned to the improved performance of frequency response [11]. The main challenges facing de-loading and KE extraction are continuous curtailment of wind power and post-event recovery respectively. The amount of non-supplied energy due to de-loading techniques could be estimated according to the expected load curtailments. These curtailments will have an evident impact on frequency response as well as the financial aspects of system operation and dispatching. Simplified mathematical representation of the power system could be applied to calculate analytically the system inertia and available primary reserve of WFs [12]. Meanwhile, the recovery stage (i.e. post 
frequency drops declared clear) could trigger further frequency drops, as the WTG output is suddenly reduced to start the recovery process, which is a key challenge of KE extraction. This could be mitigated by applying a pre-set shaping function with a ramp reduction in the WTG electrical output. The shaping function was triggered automatically independent of the drop severity when the frequency violated the safe deadband to initiate a step increase in the reference power signal of the WTG [13]. The technology and research challenges classification in considered six main categories of exploitation: frequency deadband, RoCoF, droop control, de-loading parameters, wind turbine level and wind farm-wide. However, the generator technology was not included which identify this area as a knowledge gap that could be tackled by this paper. The same reference has also presented the common designs of the supplementary controls used to apply the different concepts of frequency support, and their integrative approach to the holistic controls of the WTG [14].

The distinct advantages of high torque density, typically $30 \%$ rated power electronics, and inherently decoupled power control, have made the DFIG a widely adopted cost-effective solution for multi-MW wind turbines with restricted variable speed ranges (e.g. 2:1 or so) [15]. Nevertheless, the presence of brush gear unavoidably reduces its reliability raising the maintenance requirements, especially off-shore [16]. The BDFG overcomes the above DFIG drawbacks and has been regarded as a viable replacement. Unlike the DFIG, the BDFG has two ordinary, distributed 3-phase stator windings of different applied frequencies and pole numbers, and a rotor with half the total number of stator poles for the shaft position dependent magnetic coupling to occur between the windings, a pre-requisite for the torque production. The rotor can be of special 'nested-loop' cage or wound 
structure (e.g. BDFIG) [17], featuring rather complicated and strongly parameter reliant control, or modern cage-less reluctance form (e.g. BDFRG) [18] allowing similar control simplifications of DFIG [15]. The primary (power) winding is directly grid connected, while the secondary (control) winding is supplied through a fractional dual-bridge converter in 'back-to-back' configuration for bi-directional power flow as with the DFIG (Fig. 1).

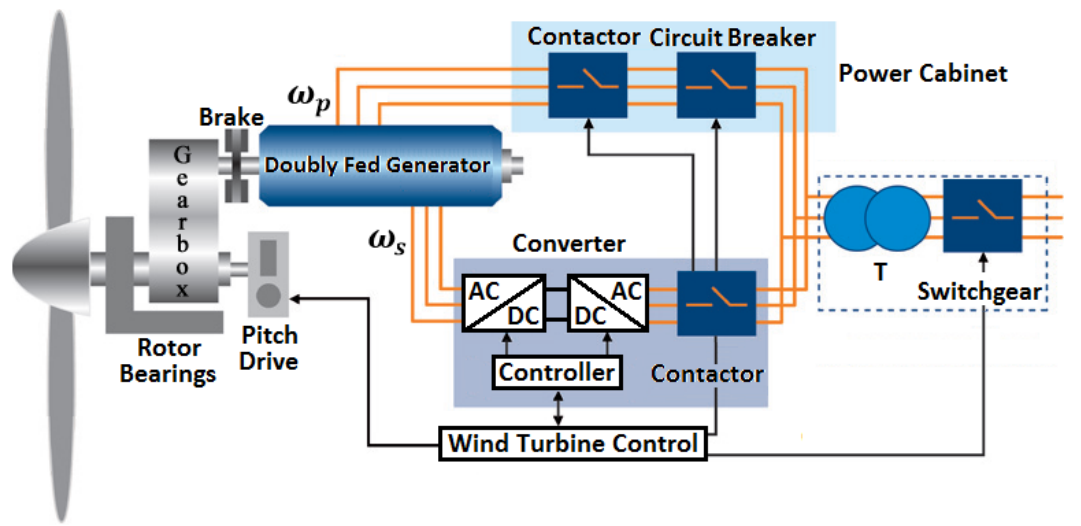

Figure 1: A generic conceptual diagram of the BDFG and DFIG wind turbine for adjustable speed constant frequency grid-connected applications ${ }^{2}$.

As the BDFG name reiterates, brushes and slip rings are eradicated, hence a more robust and maintenance-free construction. Besides, the BDFG is essentially a medium-speed machine, which renders the vulnerable high-speed stage of a three-step planetary DFIG gearbox redundant, enhancing the reliability and bringing further economic benefits [16]. These favorable properties are particularly appealing for off-shore WFs, where the DFIG running costs can be considerable [19]. Moreover, another salient BDFG merit to be pointed out is the distinguishing low-voltage-ride-through (LVRT) capability, which can be realised with much facilitated, or completely without, protective crowbar circuitry for the machine-side converter unlike the DFIG. Such attractive BDFG attributes have 
been afforded by the proportionally higher leakage inductances and lower fault current amplitudes than the equivalent DFIG having the well-known difficulties to fully satisfy the LVRT pertaining grid codes [19].

Similar research to that conducted for the DFIG [15] has been carried out on the BDFRG involving: vector control with voltage [20] or flux (field) spacevector orientation [21] including sensorless operation since an encoder is required for current control in a rotating frame, even though purely sensorless field oriented control is feasible as documented in [22] using the maximum torque per inverter ampere objective. The encoder-less BDFRG operation under power factor field oriented control conditions, as an extension of this experimental vector control research, has been elaborated in [23], direct torque [24] and power control [25]. Despite the apparent efforts and significant progress made in this direction, apart from the aforementioned large-scale design studies [26], predominantly smallscale laboratory prototyping has been the focus of attention among investigators, except for the more sizeable BDFRGs reported in [27] and the latest one elaborated in [17]. Although the BDFRG has been experimentally proven competitive with the DFIG concerning both cost and performance [28], there is very little documented on its grid integration issues and literally nothing published on the feasibility to assist in the power system frequency control. Instead, the existing literature on the subject has mainly concentrated on the established industrial technologies used for this purpose, the traditional DFIG [29] and/or the permanent magnet generator [30]. However, publications on comparative analyses of frequency support potential of these two, or any other WTGs are scarce [31].

\footnotetext{
${ }^{2}$ The control winding is on the stator for the BDFG, and on the rotor for the DFIG, but this is not detailed in the figure for convenience.
} 
Although the concepts of KE extraction and output de-loading are widely discussed in the literature, the proposed implementation methods are different where the droop de-loading is applied to manipulate linearly to the instantaneous frequency deviation. Likewise, the KE extraction is implemented through the tuning of an over-demand parameter, which influences the ratio between optimum and actual torque references. The adopted methods and parameters settings are aligned to enable a reasonable and fair comparison between the impacts of both the WTG technology and the support methods. The key parameters in both methods are selected to be equivalent, such that the de-loading and extraction factors are equal and both controllers have the same frequency deadband and frequency drop limit to release the full reserve. The applied case studies are also designed to consider and emphasize the impact of WS changes either on the dynamics of the WTG or the response of the power system, where the load dynamics are incorporated to cause fine variations on the system frequency. This examines the reactions of the WTG under like-real frequency oscillations when applying different combinations of two different generator technologies and two frequency support methods. The exploitation of these aspects is very limited in the literature. Finally, this paper presents a simplified method to estimate the dynamic inertia of the power system under the penetration of wind power. Consequently, this paper will therefore contemplate on the comparisons of the prominent and forthcoming BDFRG, and the prevailing DFIG capacities to provide such an important ancillary service operating as MW range WTGs. 


\section{BDFRG Model and Operating Aspects}

The fundamental angular velocity and mechanical power relationships for the electro-mechanical energy conversion in the machine, showing individual contributions of each winding and assuming motoring convention as default, can be written as follows [32, 33]:

$$
\begin{gathered}
\omega_{r}=\frac{\omega_{p}+\omega_{s}}{p_{r}}=\frac{\omega_{p}}{p_{r}} \cdot\left(1+\frac{\omega_{s}}{\omega_{p}}\right)=\omega_{s y n} \cdot\left(1+\frac{\omega_{s}}{\omega_{p}}\right) \\
P_{m}=T_{e} \cdot \omega_{r}=\underbrace{\frac{T_{e} \cdot \omega_{p}}{p_{r}}}_{P_{p}}+\underbrace{\frac{T_{e} \cdot \omega_{s}}{p_{r}}}_{P_{s}}=P_{s} \cdot\left(1+\frac{\omega_{p}}{\omega_{s}}\right) \\
k=\frac{\omega_{\max }}{\omega_{\min }}=\frac{\omega_{p}+\Delta \omega_{s}}{\omega_{p}-\Delta \omega_{s}} \Longrightarrow \Delta \omega_{s}=\frac{k-1}{k+1} \cdot \omega_{p}
\end{gathered}
$$

where $\omega_{\text {syn }}=\omega_{p} / p_{r}[\mathrm{rad} / \mathrm{s}]$ is obtained for $\omega_{s}=0$ i.e. a DC secondary as with a $2 p_{r}$-pole wound field synchronous turbo-machine, $\omega_{s}>0$ for 'supersynchronous' operation, and $\omega_{s}<0$ (e.g. an opposite phase sequence of the secondary to the primary winding) in 'sub-synchronous' mode. Therefore, for the same number of rotor poles, the synchronous speed $\left(\omega_{\text {syn }}\right)$ of the BDFRG is half that of the DFIG, which implies that the 3rd stage of a gearbox, prone to high failure rates and hence expensive repairs (foremost at sea), can be avoided improving the reliability and reducing the WTG downtime as well as the maintenance costs [16]. In the BDFRG case, $T_{e}<0$ and thus $P_{p}<0$ in (2), so that the positive power is actually fed to the grid by the primary winding as expected in the generating mode, while the secondary power $\left(P_{s}\right)$ flow can be bi-directional subject to the operating speed region as depicted in Fig. 2. Note also that if the desired operating speed range is $2: 1$ i.e. $\pm \Delta \omega_{s} / p_{r}$ around $\omega_{\text {syn }}$, being common for WTGs, then $k=2$ in (3) and the maximum secondary frequency turns out to 


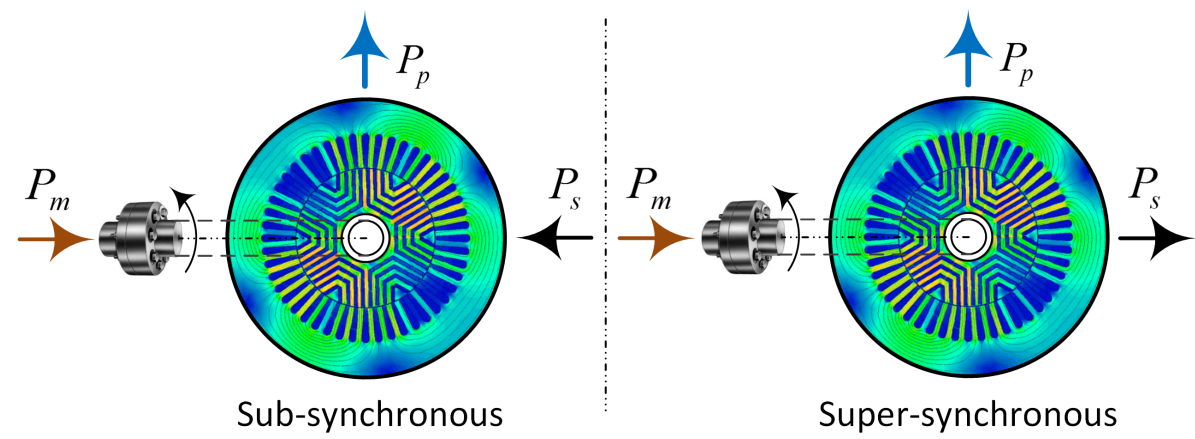

Figure 2: Reference directions of real power flow in the BDFRG windings for the operation below and above the synchronous speed.

200

be $\Delta \omega_{s}=\omega_{p} / 3$ whereas $P_{s}=P_{m} / 4$ according to (2). This means that only a $25 \%$ rated converter would be ideally needed as with the DFIG wind turbines.

The BDFRG dynamic and steady-state models in a $d_{p}-q_{p}$ frame for the $\omega_{p}$ rotating primary winding space-vectors, and a $d_{s}-q_{s}$ frame for the $\omega_{s}$ rotating secondary counterparts (Fig. 3) can be represented as [20-23, 34]:

$$
\begin{gathered}
\underline{v}_{p}=R_{p} \underline{i}_{p}+\frac{d \underline{\lambda}_{p}}{d t}=R_{p} \underline{i}_{p}+j \omega_{p} \underline{\lambda}_{p} \\
\underline{v}_{s}=R_{s} \underline{i}_{s}+\frac{d \underline{\lambda}_{s}}{d t}=R_{s} \underline{i}_{s}+j \omega_{s} \underline{\lambda}_{s}
\end{gathered}
$$

$$
\left.\begin{array}{l}
\underline{\lambda}_{p}=L_{p} \underbrace{\left(i_{p d}+j i_{p q}\right)}_{\underline{i}_{p}}+L_{m} \underbrace{\left(i_{s m_{d}}-j i_{s m_{q}}\right)}_{\underline{i}_{s m}^{*}} \\
\underline{\lambda}_{s}=L_{s} \underbrace{\left(i_{s d}+j i_{s q}\right)}_{\underline{i}_{s}}+L_{m} \underbrace{\left(i_{p m_{d}}-j i_{p m_{q}}\right)}_{\underline{i}_{p m}^{*}}
\end{array}\right\}
$$




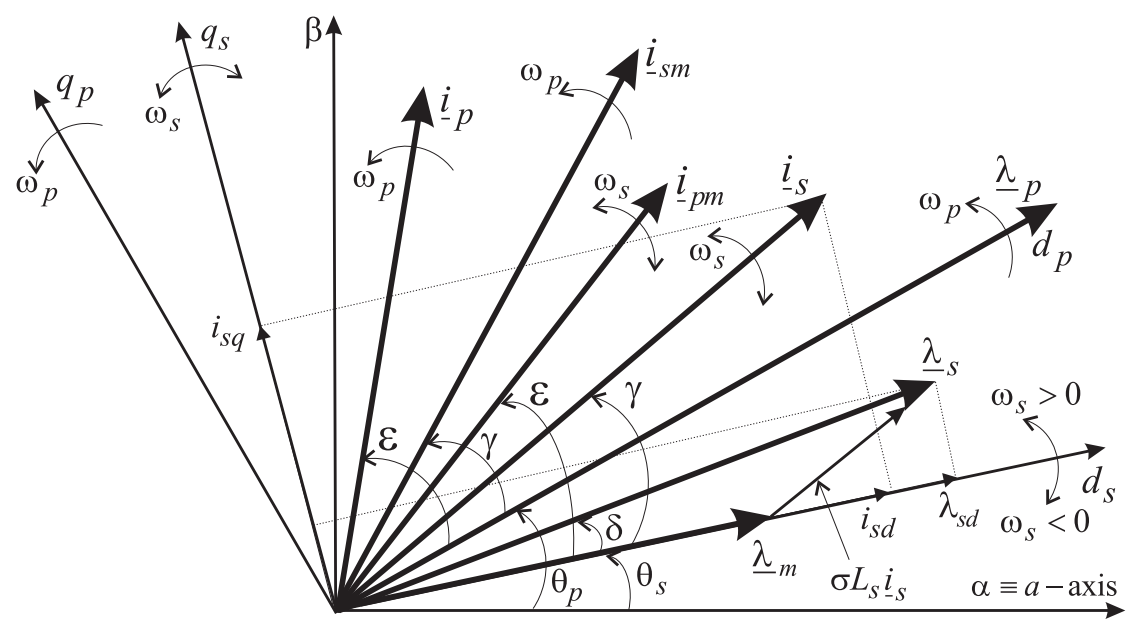

Figure 3: Characteristic space vectors and flux-oriented reference frames relevant for the BDFRG dynamic modelling and control.

The flux equations of (4) can be manipulated as [20-23, 34]:

$$
\begin{aligned}
& \underline{\lambda}_{p}=\underbrace{L_{p} i_{p d}+L_{m} i_{s m_{d}}}_{\lambda_{p d}}+j \cdot \underbrace{\left(L_{p} i_{p q}-L_{m} i_{s m_{q}}\right)}_{\lambda_{p q}} \\
& \underline{\lambda}_{s}=\underbrace{\sigma L_{s} i_{s d}+\lambda_{m d}}_{\lambda_{s d}}+j \cdot \underbrace{\left(\sigma L_{s} i_{s q}+\lambda_{m q}\right)}_{\lambda_{s q}}=\sigma L_{s} \underline{i}_{s}+\underbrace{\frac{L_{m}}{L_{p}} \lambda_{p}^{*}}_{\underline{\lambda}_{m}}
\end{aligned}
$$

where $\lambda_{m}$ is the primary flux coupling the secondary winding, $L_{p, s, m}$ are the 3phase self and mutual inductances derived in [32,33], $\sigma=1-L_{m}^{2} /\left(L_{p} L_{s}\right)$ is the leakage factor, $\underline{i}_{s m}$ is the magnetically coupled (magnetizing) secondary current vector $\left(\underline{i}_{s}\right)$ of the same magnitude but modulated frequency (i.e. $\underline{i}_{s m}=\underline{i}_{s}$ in the respective frames), and vice-versa for $\underline{i}_{p m}=\underline{i}_{p}$ as shown in Fig. 3. Notice that $\underline{i}_{s m}, \underline{i}_{p}$ and $\underline{\lambda}_{p}$ in (4) and (5) are in the $\omega_{p}$ frame, whereas $\underline{i}_{s}, \underline{i}_{p m}$ and $\underline{\lambda}_{m}$ in (4) and (6) are in the $p_{r} \omega_{r m}-\omega_{p}=\omega_{s}$ frame given (1). This frame selection is termed as 'natural' since the corresponding $d q$ vector components become DC, which are 
easier to control. The remaining dynamic modeling and operating principles of the BDFRG are detailed in $[32,33]$.

It is interesting that regardless of the unconventional machine design, entirely different basic theory and unusual torque producing mechanism $[32,33]$, the BDFRG model described by (4)-(6) in many respects resembles that for the DFIG [15]. In fact, the BDFRG primary and secondary windings, and the associated quantities, play the roles of the DFIG stator and rotor equivalents, respectively. However, the awkward parameter referencing (Fig. 3) and other pertinent structural complexities make the BDFRG model much more challenging to implement and simulate. In addition, the intrinsically modest magnetic coupling (e.g. higher $\sigma$ values) may compromise the BDFRG transient response to some extent relative to DFIG.

\section{Frequency Support Methods and Operation}

\subsection{Pitch Droop De-loading}

This technique can either be applied as Delta de-loading, when the WTG output is de-rated continuously with a fixed relative ratio from the available power, or Balance de-loading where an immobile margin between the MPT operation (e.g. without frequency regulation) and the actual outputs is kept throughout to specify the de-rating profile of interest [35]. This section is concerned with the implementation of the Delta concept in which the reference pitch angle is varied to maintain a predetermined de-loading ratio $\left(D_{F}\right)$ and preserve the nominal rotor speed for a given WS. The active power reference $\left(P_{r e f}^{o}\right)$ is decreased by the required $D_{F}$ according to Fig. 4, and the latter is curtailed regularly through a droop gain until the frequency drop reaches a predefined threshold $\left(f_{d r o p_{\max }}\right)$, as illustrated by the 

$=15 \%, f_{\text {drop } \max }=49.5 \mathrm{~Hz}$, and $f_{\text {low }}=49.95 \mathrm{~Hz}$.
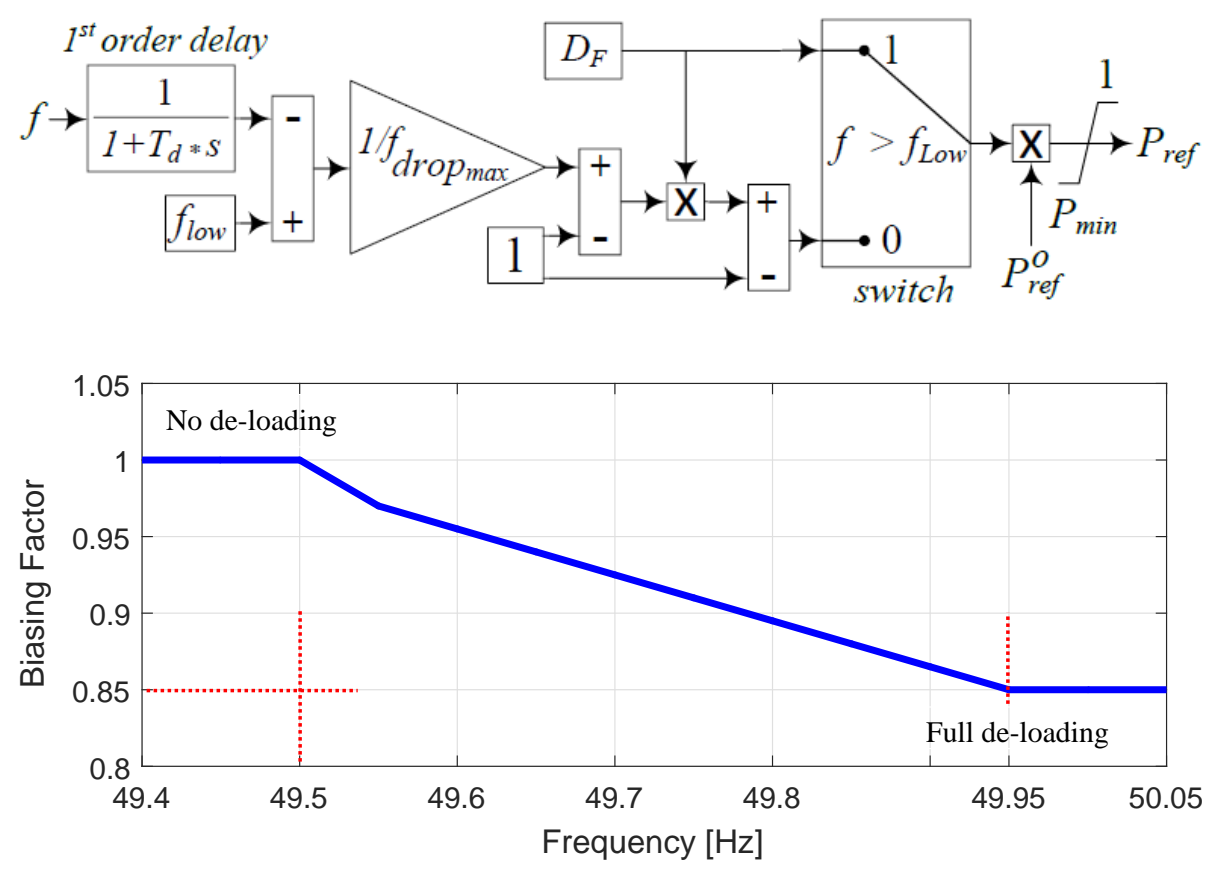

Figure 4: Supplementary controller diagram and curtailment pattern of per unit de-loading based on the incident frequency drop.

supplementary controller design in Fig. 4. For example, when the frequency violates a certain lower limit $\left(f_{\text {low }}\right)$, the WTG output could be as depicted, where $D_{F}$

Such a procedure smoothens the frequency response and mimics the governor droop behaviour of conventional SGs. The de-loading is deactivated at higher WSs, when the WTG delivers its rated power to mitigate the lost energy. However, pitching is normally enabled to keep the WTG output and the rotor speed within the acceptable limits, but at frequency incidents, the pitch angle can be reduced to allow a temporary WTG overload, which must be compliant with the manufacturer specifications to avoid excessive over-heating or other potentially damaging circumstances for the machine [8]. 


\subsection{Kinetic Energy Extraction}

This approach may be a preferable choice since it does not imply any energy losses for the normal MPT operation of WFs without frequency control. However, for the same WS, the power surge is lower compared to the de-loading method, and there is also high susceptibility to WS conditions both during and after the frequency event. The scheme relies on the rise of reference torque $\left(T_{r e f}\right)$ beyond its nominal value $\left(T_{r e f}^{o}\right)$. Hence, the higher electrical demand than the available mechanical input forces the WTG to extract some of its KE in order to secure the necessary power support. The extraction factor $\left(K_{e x}\right)$ is governed by the severity of the frequency drop through a droop response by analogy to the de-loading method ( $\left.1 \leq K_{e x} \leq 1.15\right)$ as shown in Fig. 5. The KE extraction process halts

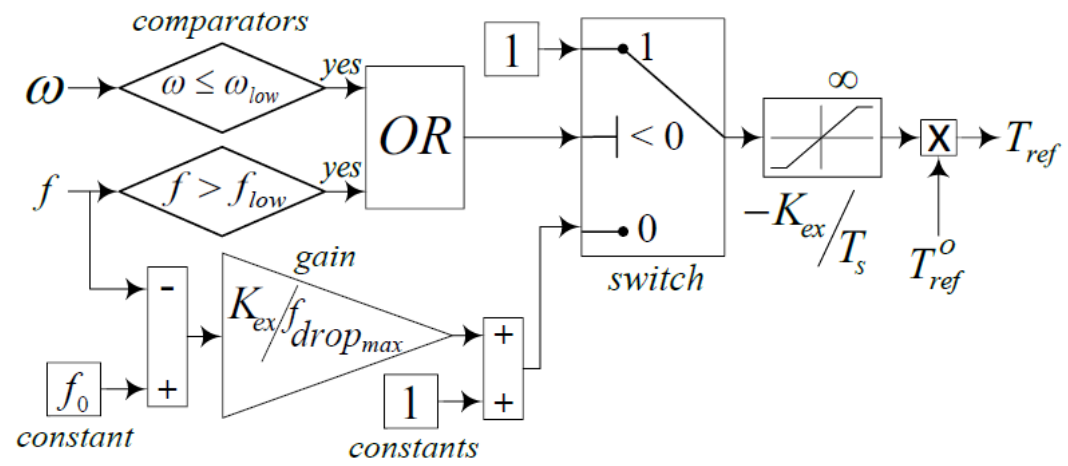

Figure 5: Subsidiary controller schematic of the KE extraction method.

when the rotor speed $(\omega)$ reaches a predefined lower threshold $\left(\omega_{\text {low }}\right)$. This method is disabled in case of severe WS drops, which are unlikely to happen, as there is no back-up source of energy to maintain the supportive power surge, and it also puts the WTG under the risk of complete halt. The post-event stage is also critical where the WTG accelerates to recover the nominal rotor speed, implying a reference torque that is less than the nominal value. 

of the two WTG technologies can be made.

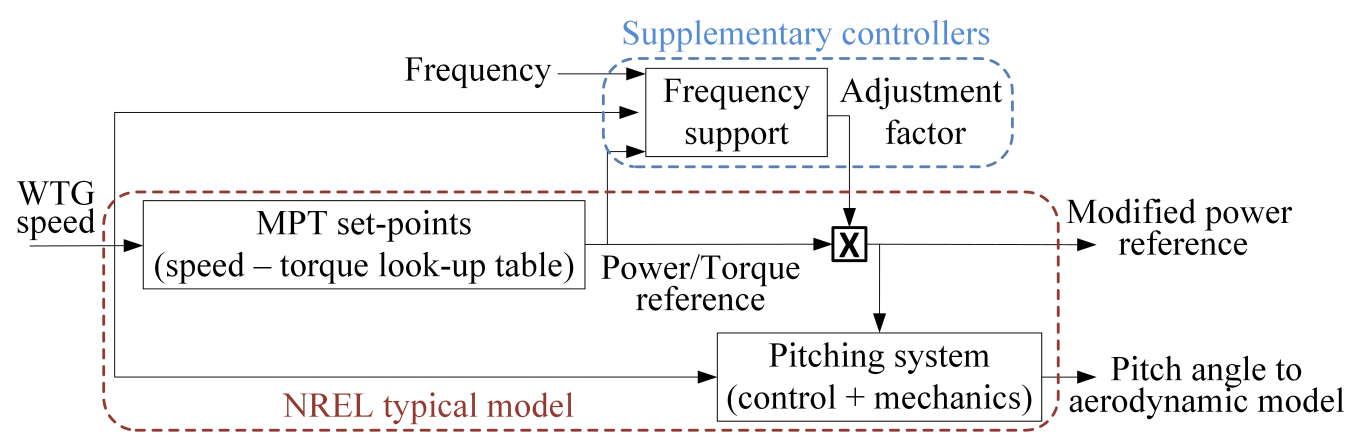

Figure 6: A generic block diagram of the upgraded NREL ${ }^{\circledR}$ WTG model [36] with the integrated supplementary frequency controllers.

\subsection{WTG Modeling and Control}

The auxiliary control algorithms from Figs. 4 and 5 are incorporated into the realistic detailed DFIG (Type 3) NREL ${ }^{\circledR}$ model from the Simulink ${ }^{\circledR}$ library as outlined in Fig. 6 [36]. The replica dynamic model for an equally rated BDFRG with independent real and reactive power control similar to DFIG [20,34], and an optimised 'ducted' rotor design (Fig. 2) [18, 26, 37], has been built by employing the same $\mathrm{GE}^{\circledR}$ wind turbine so that useful comparative performance evaluations

The developed supplementary controllers are also suitably configured to allow a fair comparison between the underlying frequency support concepts using the measured frequency input signals to trigger control actions. Furthermore, the other key parameters in both methods are likewise selected to be identical for either WTG. For example, the de-loading and extraction factors are equal, and both controllers have the same frequency drop dead-bands and limits to release the full reserve. The WTG specifications and per-unit values used for the simulation analyses are summarised in Table 1. 
Table 1: The parameters of the DFIG and BDFRG wind turbines

\begin{tabular}{lll}
\hline \hline Parameter & DFIG & BDFRG \\
\hline Rated power (MW) & 1.5 & 1.5 \\
Line voltage (V), frequency (Hz) & 690,50 & 690,50 \\
Rated speed (rev/min) & 1800 & 600 \\
Stator, rotor poles & 4,4 & $8 / 4,6$ \\
\hline Power winding resistance (pu) & 0.0073 & 0.022 \\
Power winding leakage inductance (pu) & 0.1766 & 0.2519 \\
Control winding resistance (pu) & 0.0052 & 0.0446 \\
Control winding leakage inductance (pu) & 0.1610 & 1.1021 \\
Magnetizing (mutual) inductance (pu) & 2.9913 & 4.4084 \\
\hline Rated wind speed (m/s) & 13 & 13 \\
Gearbox stages/ratio & $3 / 90$ & $2 / 30$ \\
Drive-train inertia constant (s) & 4.74 & 5.2 \\
\hline \hline
\end{tabular}

Note, however, that while the frequency regulation strategies under consideration have been intensively investigated and applied in the literature [38], whereby the corresponding implementation procedures used in this paper are inevitably different when the droop de-loading ratio is manipulated in response to the frequency deviation as shown in Fig. 4. Besides, the KE extraction is achieved by appropriate tuning of the over-demand parameter $\left(K_{e x}\right.$ in Fig. 5), which influences the ratio between the optimum and actual torque references.

\section{Studies of Wind Speed Effects}

The following subsections examine a frequency scenario for step-changes of average WS and realistic wind profiles, and the response of the proposed methods and machines to a certain frequency test signal. The WS fluctuating nature is a 
major challenge facing wind energy as a provider of frequency support, hence it is important to exploit its impact when WS changes while the WTG/WF is providing frequency support. The impact of WS is also relevant to the comprehensive comparison between the two machines where the input mechanical power changes according to incident WS. Moreover, the proposed controls have certain modes of operation to mitigate the negative influence of WS steep drops on the provided support. Therefore, this paper tests the proposed methods and machines under a steep WS drop simultaneously with a frequency event. In this section, a test frequency signal is applied to the controls of the WTG, where the frequency drops by $f_{\text {drop } \max }=0.5 \mathrm{~Hz}$ from its nominal value $\left(f_{o}\right)$ linearly within $1 \mathrm{~s}$ as shown in Fig. 7, which means that the RoCoF is $-0.5 \mathrm{~Hz} / \mathrm{s}$.

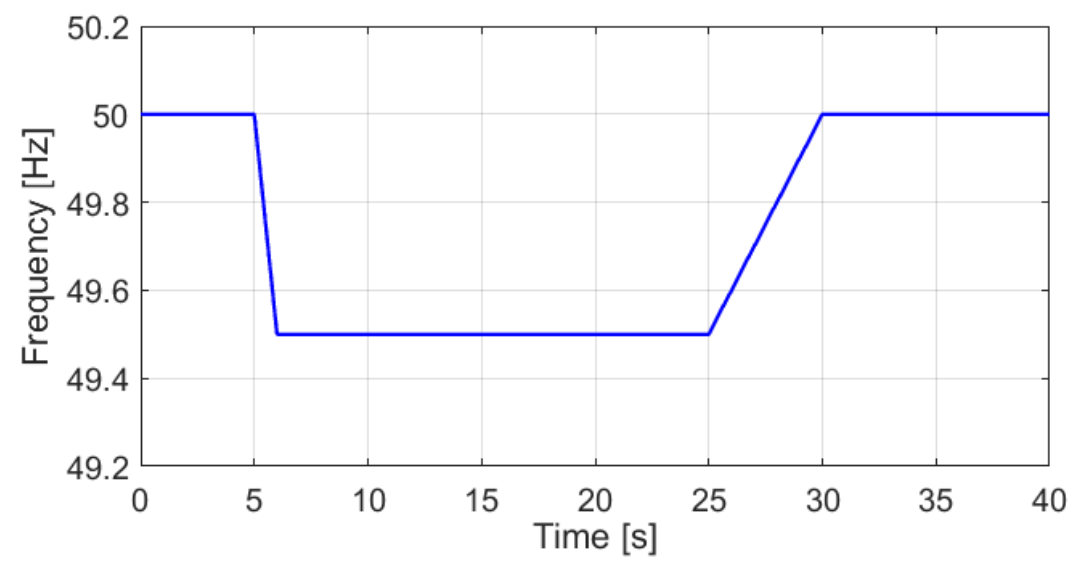

Figure 7: The applied frequency test signal complementary to Fig. 8 and Fig. 9.

This is inspired by the common requirements of grid codes [39]. Afterwards, at $\mathrm{t}=25 \mathrm{~s}$ the frequency gradually recovers to its nominal value with a rate of change of $0.1 \mathrm{~Hz} / \mathrm{s}$. Thus, the WTGs response to frequency dips, and not minor oscillations caused by mild load variations, is one of the main concerns of this 
work. In order to enable better capturing of the WTG dynamics even under turbulent winds, the sampling rate of $1 \mathrm{~s}$ is chosen for the applied intermittent WS conditions.

\subsection{Step Responses}

Two sudden WS changes are initiated at $t=15 \mathrm{~s}(\mathrm{e} . \mathrm{g}$. from $8.2 \mathrm{~m} / \mathrm{s}$ to $7.1 \mathrm{~m} / \mathrm{s}$ ) and $\mathrm{t}=22 \mathrm{~s}$ (e.g. a rise of $0.5 \mathrm{~m} / \mathrm{s}$ ). The power waveforms in Fig. 8 confirm that the normal (i.e. no frequency event) WTG output is reduced in case of the deloaded method, while the KE extraction counterpart follows the MPT trajectory providing a sustained power surge. The worst power dip occurs after the stored $\mathrm{KE}$ is exhausted with the WTG speed reaching its lower threshold as in Fig. 8. The zoomed image frames are integrated to the main figures to enhance the visualization of some reflective dynamics in order to evidence the differences between the waveforms amongst the two WTGs without compromising the visuals of the full simulation span.

The WS drop makes the WTGs to temporarily divert away from the nearly nominal speed as illustrated in Fig. 8. During the frequency recovery, it takes about $25 \mathrm{~s}$ for the WTGs to regain the inceptive steady-state conditions due to the inertia of the rotating masses, and the increasing pitch angles required to restore the de-loaded operation. On the contrary, the KE extraction method does not imply such long transients with relatively high speed deviations for modest WS fluctuations, mainly because the pitch angle is not deployed. The speed recovery relies on the WS prior to the support provision and the attained drift during the frequency event, where it reaches $0.63 \mathrm{pu}$. In spite of the continuous variations in the available wind power, which are largely dictated by the WTG aerodynamics and rotor speed controllers, the electrical torque $\left(T_{e}\right)$ accurately follows the me- 

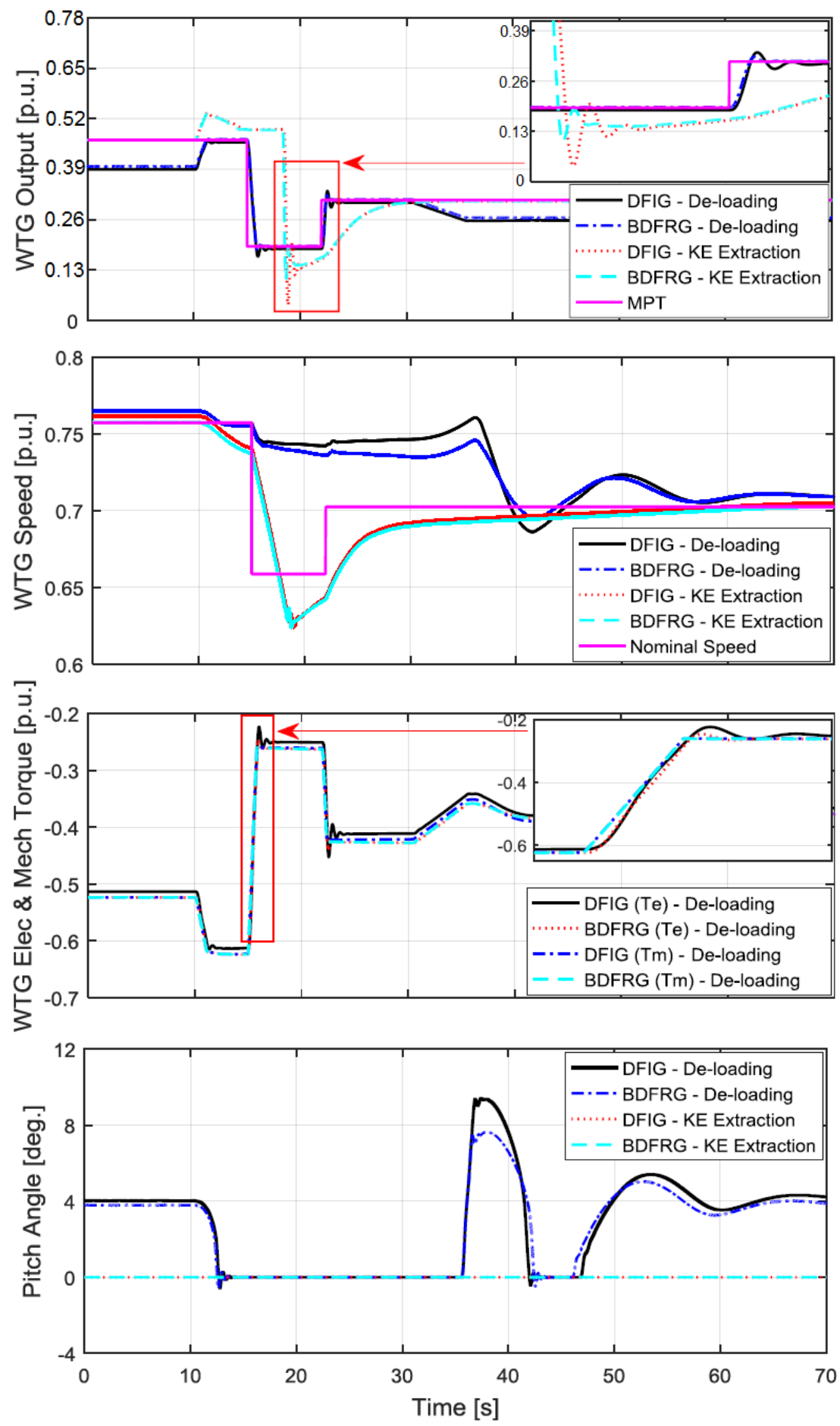

Figure 8: WTGs response to WS step changes for the two support methods. 
chanical $\left(T_{m}\right)$. A quick torque response to the very steep change induced by the sudden WS reduction is noticeable from the respective sub-plot in Fig. 8. It is also visible that a generally good matching of the torque waveforms is slightly better for the BDFRG, while the WTGs experience minor oscillations before reaching a new steady-state point with the de-loading method being applied.

The results in Fig. 8 suggest a very close overall performance of the two WTGs, the BDFRG exhibiting a marginally slower response when the pitch angle is recovering to its non-zero value after the frequency transient. This is manifested through the WTG speed under the de-loading method, and it is believed to predominantly come from the somewhat higher inertia of the BDFRG drive train. To achieve the DFIG de-loading level, a lower pitch angle is needed for the BDFRG (e.g. the respective peak values are $\approx 7^{\circ}$ and $10^{\circ}$ ), which may count as a merit since the mechanical stresses exerted on the pitching mechanism should be reduced as much as possible.

In summary, the sustainability of the WTG output under the KE extraction method conditions is highly related to the initial WS and the WTG inertia. Conversely, the de-loading strategy does not imply any recovery periods, but the output reduction process is naturally smooth via pitch control effects. In addition, the applied droop pattern ensures the return to the normal de-loaded operation without negative implications.

\subsection{Natural Wind Speed Profile}

True WS measurement data with an average of about $8 \mathrm{~m} / \mathrm{s}$ are used to investigate the comparative WTGs performance under the intermittent WS conditions. The WTG output characteristics presented in Fig. 9 are generally sub-optimal for the de-loading method, however, the desired de-loading ratio is not fully achieved 
as the pitch controller is not able to track all the intended WS fluctuations. Implementing the support method makes the WTG to attain the available MPT output. The KE extraction technique has proven capable of providing sustainable support (but with a lower magnitude compared to the de-loading method at medium and high WSs) as highlighted in Fig. 9. The additional power surge is kept constant until the depletion of the stored KE stops to avoid the WTG deceleration, evidenced by the decreasing speed plots in the same figure, due to the imbalance between the turbine $\left(T_{m}\right)$ and generator $\left(T_{e}\right)$ torque. The amount of supportive energy is higher than with the de-loading method, because the WS drop emphasises the benefit of the KE extraction approach. The intermittent WS profile screens the dynamics of both machines, hence their very similar responses. It is also shown that a large inertia and slow responses curtail the WTG ability to match the optimum rotor speed for a given WS at each time step. However, the deceleration impact caused by the KE extraction method is evident during the frequency transient and as a consequence the WTG attempts to maintain a fixed power surge according to Figs. 4 and 5.

The results in Fig. 9 of comparing the $T_{e}$ variations of the two generator technologies and the two support methods reflect the minor deviations between the two generators. However, when the WTG reaches the threshold rotor low speed which halts the KE exctraction process, the DFIG experiences a sharper transient response compared to BDFRG, which is further clarified through the employed zoom in frame. It can be also noticed the steeper tranisents in case of droop pitch de-loading compared to KE extraction method due to the continous variations of reference pitch angle to maintain the required de-loading ratio. Meanwhile, the responses for either WTG are nearly overlapping. The pitch angle dynamics con- 

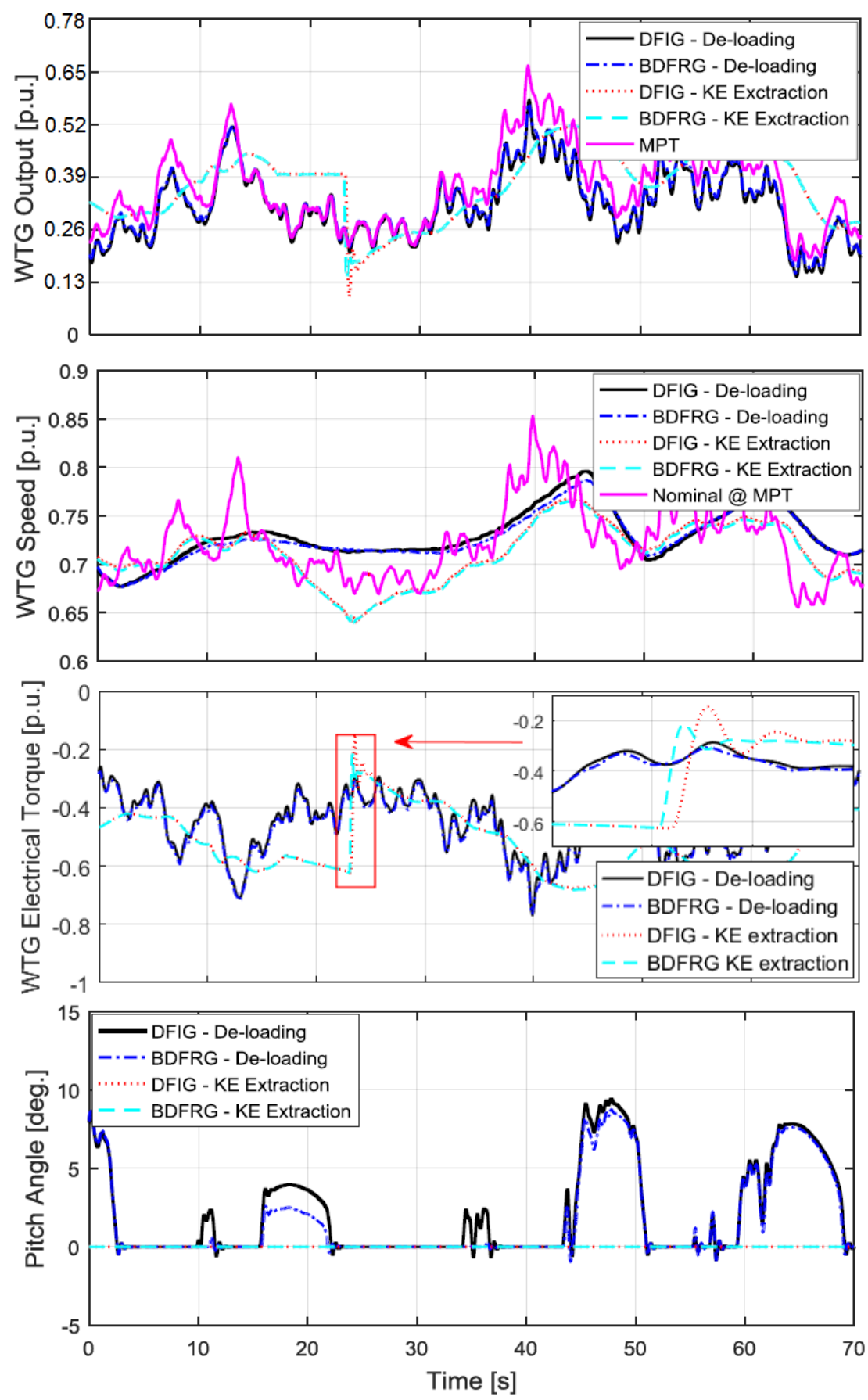

Figure 9: WTGs response provided by the two support methods for a realistic wind speed profile. 
firms that the de-loading exerts higher stresses on the WTG pitching system than the KE extraction method, which does not require any additional pitching and where at an average WS, the pitch angle is zero at all times. The BDFRG ensures slightly reduced pitch angle changes than the DFIG by analogy to the previous case study in Section 4.1.

\section{Analysis of Power System Frequency Response}

The considered benchmark is composed of an aggregate synchronous area and installed conventional generation capacity $\left(P_{c}^{o}\right)$ feeding a composite load as shown in Fig. 10. The AC area inclusion is intended to adequately represent a medium scale power network with an average vulnerability to the frequency fluctuations, which can be modelled as [29]:

$$
\begin{aligned}
\dot{f} & =\frac{P_{c}+P_{W F}-P_{L}-\left(f-f_{o}\right) \cdot\left(D_{l}+4 D_{g} f \pi^{2}\right)}{4 J f \pi^{2}} \\
\dot{P}_{c} & =\frac{P_{c}^{o} \cdot\left[\text { Loading }-\left(f-f_{o}\right) /\left(R f_{o}\right)\right]-P_{c}}{T_{g e n}}
\end{aligned}
$$

where $P_{c}$ and $P_{W F}$ are the actual conventional and wind power generation, respectively, $P_{L}$ is the total main load and Loading (e.g. 95\% of $P_{c}^{o}$ in this paper) signifies the $P_{c}$ output set-point under normal (pre-event) operating conditions (i.e. $5.32 \mathrm{GW}$ ), whereas $f_{o}$ is the nominal grid frequency (e.g. 50-Hz). The wind installed capacity is assumed to be $35 \%$ of $P_{c}^{o}$ with a $50 \%$ WF capacity factor.

The WF is represented by an aggregate WTG of an equivalent capacity of the WF, and facing a unified WS pattern. This does not compromise the level of details of the applied test system as the aggregate models include the essential electromechanical components of the wind energy system. This aggregation 


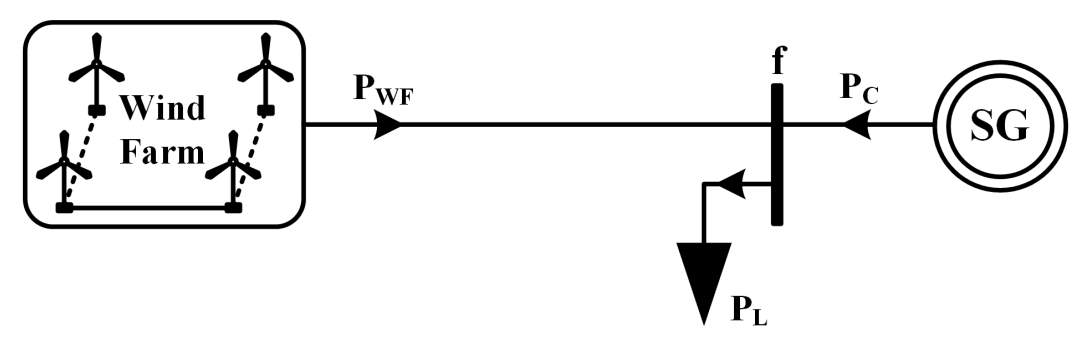

Figure 10: Conceptual representation of the implemented benchmark.

approach is used to achieve relatively high penetration level of wind power into the test power system avoiding the additional computational efforts to model and simulate each wind turbine and its incident wind speed separately. The remaining model specifications are enlisted in Table 2. To retain consistency throughout the paper authors have opted that the inertia constant in these units to comply with the detailed modelling of the synchronous AC area which is described in (7) and (8), thus inertia of the system in seconds is analysed in the next section.

Table 2: The parameters of the aggregate synchronous area

\begin{tabular}{ll}
\hline \hline Conventional generation capacity $\left(P_{c}^{o}\right)$ & $5.6 \mathrm{GW}$ \\
Generation time constant $\left(T_{g e n}\right)$ & $2 \mathrm{~s}$ \\
Lumped moment of inertia $(J)$ & $0.39 \mathrm{M} \cdot \mathrm{kg} \cdot \mathrm{m}^{2}$ \\
Generation droop $(R)$ & 0.06 \\
Damping ratio $\left(D_{g}\right)$ & 95 \\
Load frequency sensitivity ratio $\left(D_{l}\right)$ & $1 \%$ \\
\hline \hline
\end{tabular}

The system dynamic inertia constant $\left(H_{d}\right)$ is estimated during the critical stages of the frequency event using the following expression:

$$
H_{d}=\frac{f_{o}}{2 \dot{f}} \cdot \frac{\Delta P}{P_{c}+P_{W F}}
$$


providing that $\dot{f} \leq-0.1 \mathrm{~Hz} / \mathrm{s}$ and $f<f_{o}$ with $\Delta P$ denoting the instantaneous mismatch between generation and demand. The dynamic inertia is actually the inertia constant of the system captured at different time points during the frequency event. The system inertia under high penetration of wind energy is highly variable, so it is indicative to analyse the impact of WFs a provider of frequency support on system inertia including the turbine WTG technology and the adopted support method. This confirms the reasonable and expansive nature of the presented study in this paper, where $\left(H_{d}\right)$ is calculated using (9) given that the system frequency is outside the deadband and experiencing a high rate of decay of $0.2 \mathrm{~Hz} / \mathrm{s}$ or worse.

\subsection{Considered Operating Scenarios}

In each of the five case studies being examined, the system has suffered a serious frequency drop caused by a sudden loss of $12 \%$ of the conventional generation capacity, namely $672 \mathrm{MW}$ at $\mathrm{t}=10 \mathrm{~s}$ time instant. The lost generation is gradually recovered at $\mathrm{t}=40 \mathrm{~s}$ with a gradient of $2 \%$ per second to mimic the secondary response of SGs, and to test the compatibility of the applied support methods when the frequency rebounds to the safe margin. In order to isolate the effects of the support method and the type of machine, the WS is assumed constant at around $8 \mathrm{~m} / \mathrm{s}$ during the frequency event. This assumption is acceptable because the frequency transients should normally last no more than 10-30 s according to most grid codes [3]. Over such a short interval, and due to the heavy WTG inertia, many studies are based on the average WS conditions [40].

\subsection{Results and Discussions}

Fig. 11 shows that the frequency nadir has improved by about $0.25 \mathrm{~Hz}$ for the de-loading method and $0.15 \mathrm{~Hz}$ for the $\mathrm{KE}$ extraction counterpart. It can also be 
seen that the superior performance can be achieved with the de-loading approach as the down-pitching recaps the higher output for a power surge compared to the extracted energy at the selected $K_{e x}$. The frequency excursion clearance after the recovery of the lost generation is smooth without any spikes or overshoots due to the developed droop controller action, which regulates the supportive power surge through the droop constants (i.e. $D_{F}$ and $K_{e x}$ ) based on the frequency drop severity. It is worth mentioning that the provided WF support reduces the RoCoF during the critical early stage of the event. This is important in order to avoid any unnecessary tripping of the RoCoF relays of SGs if the pre-set threshold (e.g. typically from $0.5 \mathrm{~Hz} / \mathrm{s}$ to $1 \mathrm{~Hz} / \mathrm{s}$ during the first $500 \mathrm{~ms}$ ) is violated according to the grid code [39].

Differences brought by the two WTGs are fairly small because of the predominant power system dynamics over the WTG mechanical and aerodynamics effects. The WTG decelerates and recovers to the nominal speed at lower rates using the BDFRG, which coincides with the results obtained in Section 3. However, the maximum rotor speed deviation from its nominal value does not exceed $2 \%$. The BDFRG appears to be more resistant to speed changes in case of droop de-loading, which counts as a merit in its own right.

The WTGs power surges are almost identical for the two methods. Still, the DFIG appears to be advantageous in this sense as detailed in the zoomed window of the power plot in Fig. 11. The WTG decelerates during the KE extraction until reaching a lower speed, as depicted in Fig. 11, which corresponds to the incident frequency variation given the predetermined value of $K_{e x}$. In this case study, the frequency drop is less than $f_{\text {drop } \max }$, which helps the WTG not to attain the minimum allowed rotor speed. Hence, the WTG continues to operate at the lower 

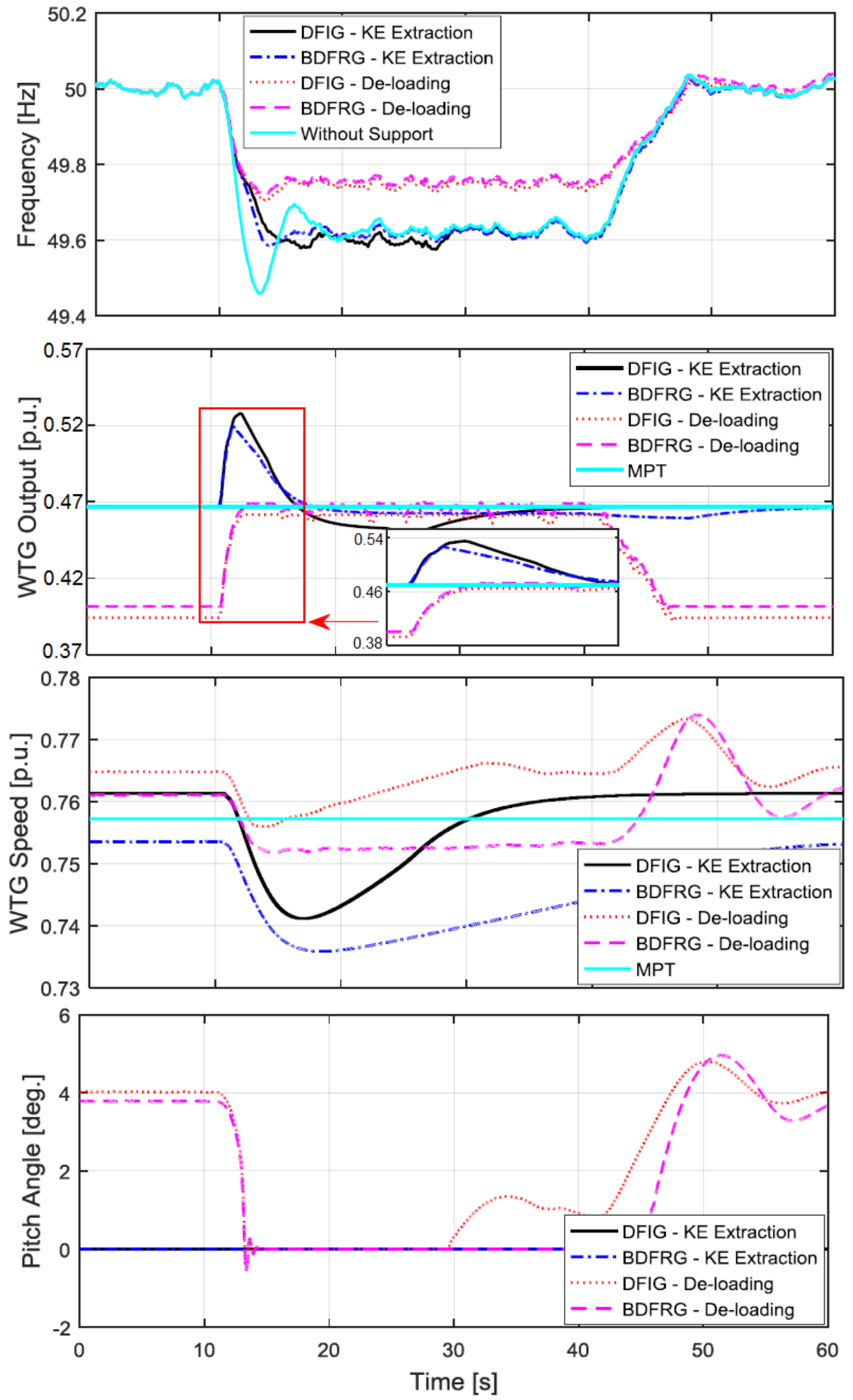

Figure 11: WTGs response using the proposed frequency support methods at fixed wind speed. 
speed without the need to recover until the frequency starts building up to reach the safe margin. The power surge sustained for about $10 \mathrm{~s}$, and the recovery to the nominal speed was slower for the BDFRG. The de-loading technique shows a little impact on the WTG speed due to the WTG aerodynamics, mostly during the pitching down as a small portion of the injected power accelerates the WTG, this being limited in the BDFRG case. On the other hand, the DFIG pitching is ahead of the BDFRG's as shown in Fig. 11.

The $H_{d}$ changes during the considered frequency scenario are presented in Fig. 12. It can be seen that the $H_{d}$ estimates have somewhat increased in all the cases. The BDFRG achieved an extended rise of about $0.4 \mathrm{~s}$ relative to the DFIG, which could be explained by its slightly faster response to the $T_{m}$ variations through the pitching process. Generally, the $H_{d}$ improvements are marginal (i.e. $0.1 \mathrm{~s}$ to $0.3 \mathrm{~s}$ ) during the critical period from $\mathrm{t}=10 \mathrm{~s}$ up to $17 \mathrm{~s}$. The frequency starts building up to its nominal value at $\mathrm{t}=40 \mathrm{~s}$ (Fig. 11), when the inertia drops by $0.15 \mathrm{~s}$ at $\mathrm{t}=44 \mathrm{~s}$ as zoomed in the right-sided window of Fig. 12, which may be attributed to the BDFRG slower rotor speed recovery and the extended time required for the WTG to reach its nominal output for a given WS. Hence, the power imbalance is diminished after a slight delay reflecting upon the $H_{d}$ reduction according to (9). However, the inertia drop is about $5 \%$ of the average value $(\approx 3 \mathrm{~s})$. In addition, the overall system inertia is reduced compared to the early stage of the event (i.e. after $t=10 \mathrm{~s}$ ).

Note that $H_{d}$ is evaluated using (9), and denoted accordingly in Fig. 12, only if there is a power mismatch (i.e. generation less than demand causing a negative RoCoF). Else, when a $H_{d}$ value is not displayed this implies an improvement as illustrated for $\mathrm{t} \in[10-15] \mathrm{s}$, where $H_{d} \approx 4 \mathrm{~s}$, while cases with frequency support 


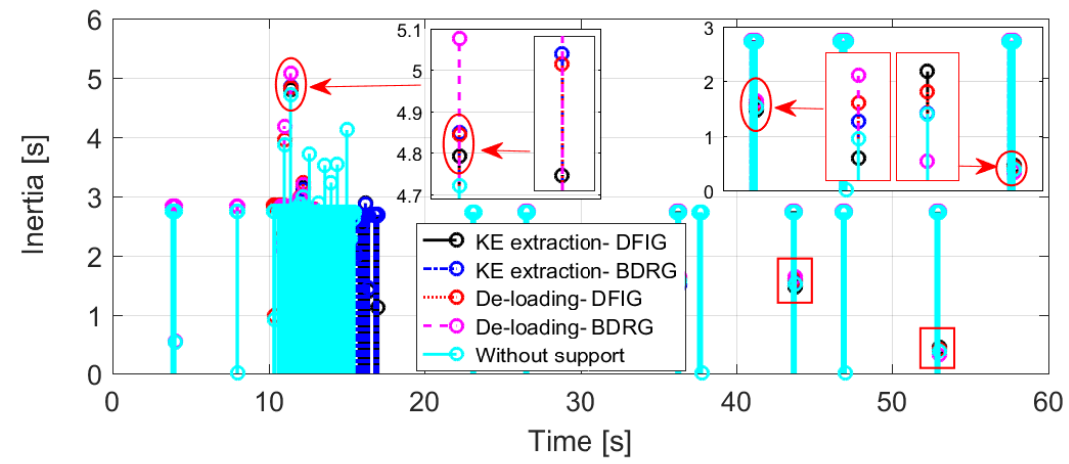

Figure 12: The system dynamic inertia constant during the early and recovery stages of the implied frequency event.

do not return a value. Conversely, at $\mathrm{t} \in[16-17] \mathrm{s}$, the KE extraction returns a relatively lower $H_{d}$, mainly for the BDFRG as discussed above, and the case without support does not give any value in this time interval.

The qualitative properties of the two WTGs in the context of frequency support provision are summarised in Table 3.

Table 3: Relative advantages (+) and limitations (-) of the WTGs

\begin{tabular}{lll}
\hline \hline Frequency Support Indicator & DFIG & BDFRG \\
\hline De-loading pitch angle & $(-)$ Higher & $(+)$ Lower \\
KE extraction rotor speed recovery & $(+)$ Faster & $(-)$ Slower \\
Extractable KE & $(-)$ Less & $(+)$ More \\
Power system dynamic inertia & $(-)$ Reduced & $(+)$ Improved \\
\hline \hline
\end{tabular}

\section{Conclusions}

This paper has made a comprehensive multi-dimensional comparison of two frequency support methods, the KE extraction and pitch de-loading, individually applied to the closely related WTG types, the conventional DFIG and evolving 
BDFRG. The major contributions of the work are therefore manifold and not only limited to comparing the integrated generator technologies for a given frequency regulation technique as its main focus, but also the other way around. The considered concepts have shown to play a pivotal role in the power injection during the frequency transient and the WTG post-incident restoration of normal operation. The KE extraction has allowed a somewhat faster DFIG recovery response than BDFRG's, while both WTGs are able to offer similar de-loading performance, with slightly mitigated pitch angle variations in the BDFRG case, which are favorable to the pitch actuators.

The frequency studies have indicated discrepancies between the WTGs, that largely depend on the ratio of the actual wind power penetration level to load demand at the inception of frequency disturbance event and the support methodology in question. The dynamic inertia evaluation has confirmed the merit of pitch de-loading approach by the absence of both adverse implications and reliance on the drive train inertia. The results presented are encouraging and have undoubtedly shown that the BDFRG can be rather competitive with the commercial DFIG of the same rating in terms of frequency support abilities to warrant further investigations as a promising brushless candidate for wind power applications.

\section{References}

[1] Y. Wang, G. Delille, H. Bayem, X. Guillaud, B. Francois, High wind power penetration in isolated power systems - assessment of wind inertial and primary frequency responses, IEEE Trans. on Power Systems 28 (3) (2013) 2412-2420.

[2] H. Banakar, C. Luo, B. T. Ooi, Impacts of wind power minute-to-minute 
variations on power system operation, IEEE Trans. on Power Systems 23 (1) (2008) 150-160.

[3] F. Diaz-Gonzalez, M. Hau, A. Sumper, O. Gomis-Bellmunt, Participation of wind power plants in system frequency control: Review of grid code requirements and control methods, Renew. and Sust. Energy Reviews 34 (2014) 551-564.

[4] M. Dreidy, H. Mokhlis, S. Mekhilef, Inertia response and frequency control techniques for renewable energy sources: A review, Renewable and Sustainable Energy Reviews 69 (2017) 144-155.

[5] A. Attya, J. Dominguez-Garcia, O. Anaya-Lara, A review on frequency support provision by wind power plants: Current and future challenges, Renew. and Sust. Energy Reviews 81 (2018) 2071 - 2087.

[6] A. D. Hansen, M. Altin, F. Iov, Provision of enhanced ancillary services from wind power plants examples and challenges, Renewable Energy 97 (2016) $8-18$.

[7] F. M. Hughes, O. Anaya-Lara, N. Jenkins, G. Strbac, Control of DFIG-based wind generation for power network support, IEEE Transactions on Power Systems 20 (4) (2005) 1958-1966.

[8] A. B. Attya, T. Hartkopf, Wind turbine contribution in frequency drop mitigation - modified operation and estimating released supportive energy, IET Generation, Transmission Distribution 8 (5) (2014) 862-872.

[9] P. Tielens, D. V. Hertem, Receding horizon control of wind power to provide frequency regulation, IEEE Trans. on Power Syst. 32 (4) (2017) 2663 - 2672. 
[10] A. B. Attya, O. Anaya-Lara, W. E. Leithead, Novel metrics to quantify the impacts of frequency support provision methods by wind power, in: IEEE PES Innovative Smart Grid Tech. Conf. Europe, 2016, pp. 1-6.

[11] F. Wilches-Bernal, J. H. Chow, J. J. Sanchez-Gasca, A fundamental study of applying wind turbines for power system frequency control, IEEE Trans. on Power Syst. 31 (2) (2016) 1496-1505.

[12] F. Teng, G. Strbac, Assessment of the role and value of frequency response support from wind plants, IEEE Trans. on Sust. Energy 7 (2016) 586-595.

[13] F. Hafiz, A. Abdennour, Optimal use of kinetic energy for the inertial support from variable speed wind turbines, Renewable Energy 80 (2015) 629 - 643.

[14] A. Aziz, A. T. Oo, A. Stojcevski, Frequency regulation capabilities in wind power plant, Sust. Energy Tech. and Assessments.

[15] R. Cardenas, R. Pena, S. Alepuz, G. Asher, Overview of control systems for the operation of DFIGs in wind energy applications, IEEE Trans. on Ind. Electron. 60 (7) (2013) 2776-2798.

[16] J. Carroll, A. McDonald, D. McMillan, Reliability comparison of wind turbines with DFIG and PMG drive trains, IEEE Trans. on Energy Convers. 30 (2) (2015) 663-670.

[17] F. Zhang, S. Yu, X. Wang, H. Wang, S. Jin, Research of a novel brushless doubly-fed generator with hybrid rotor, IEEE Trans. on Appl. Supercond. 26 (7) (2016) 1-5. 
[18] A. Knight, R. Betz, D. Dorrell, Design and analysis of brushless doubly fed reluctance machines, IEEE Transactions on Industry Applications 49 (1) (2013) 50-58.

[19] M. Cheng, Y. Zhu, The state of the art of wind energy conversion systems and technologies: A review, Energy Convers. and Manag. 88 (2014) 332 347.

[20] S. Ademi, M. Jovanovic, Control of doubly-fed reluctance generators for wind power applications, Renewable Energy 85 (2016) 171-180.

[21] S. Ademi, M. Jovanović, Vector control methods for brushless doubly fed reluctance machines, IEEE Transactions on Industrial Electronics 62 (1) (2015) 96-104.

[22] S. Ademi, M. G. Jovanović, H. Chaal, W. Cao, A new sensorless speed control scheme for doubly fed reluctance generators, IEEE Trans. on Energy Conv. 31 (3) (2016) 993-1001.

[23] S. Ademi, M. Jovanović, A novel sensorless speed controller design for doubly-fed reluctance wind turbine generators, Energy Conversion and Management 120 (2016) 229-237.

[24] H. Chaal, M. Jovanovic, Toward a generic torque and reactive power controller for doubly fed machines, IEEE Transactions on Power Electronics 27 (1) (2012) 113-121.

[25] M. Jovanović, H. Chaal, Wind power applications of doubly-fed reluctance generators with parameter-free hysteresis control, Energy Conversion and Management 134 (2017) 399-409. 
[26] D. G. Dorrell, M. Jovanović, On the possibilities of using a brushless doublyfed reluctance generator in a $2 \mathrm{MW}$ wind turbine, IEEE Industry Applications Society Annual Meeting (2008) 1-8.

[27] L. Xu, B. Guan, H. Liu, L. Gao, K. Tsai, Design and control of a highefficiency doubly-fed brushless machine for wind power generator application, in: IEEE Energy Conversion Congress and Exp., 2010, pp. 2409-2416.

[28] W. Chen, Comparison of doubly-fed induction generator and brushless doubly-fed reluctance generator for wind energy applications, Ph.D. thesis, Newcastle University, UK (2014).

[29] S. Ghosh, S. Kamalasadan, N. Senroy, J. Enslin, Doubly fed induction generator (DFIG)-based wind farm control framework for primary frequency and inertial response application, IEEE Trans. on Power Syst. 31 (3) (2016) 1861-1871.

[30] J. F. Conroy, R. Watson, Frequency response capability of full converter wind turbine generators in comparison to conventional generation, IEEE Trans. on Power Syst. 23 (2) (2008) 649-656.

[31] A. Mullane, M. O’Malley, The inertial response of induction-machine-based wind turbines, IEEE Trans. on Power Syst. 20 (3) (2005) 1496-1503.

[32] M. G. Jovanovic, R. E. Betz, J. Yu, The use of doubly fed reluctance machines for large pumps and wind turbines, IEEE Trans. on Ind. Appl. 38 (2002) 1508-1516.

[33] R. E. Betz, M. G. Jovanović, Introduction to the space vector modelling of 
the brushless doubly-fed reluctance machine, Electric Power Components and Systems 31 (8) (2003) 729-755.

[34] S. Ademi, M. Jovanović, M. Hasan, Control of brushless doubly-fed reluctance generators for wind energy conversion systems, IEEE Transactions on Energy Conversion 30 (2) (2015) 596-604.

[35] I. D. Margaris, S. A. Papathanassiou, N. D. Hatziargyriou, A. D. Hansen, P. Sorensen, Frequency control in autonomous power systems with high wind power penetration, IEEE Trans. on Sust. Energy 3 (2) (2012) 189-199.

[36] M. Singh, E. Muljadi, J. Jonkman, Simulations for wind turbine generators - with FAST and MATLAB-Simulink models, Tech. rep., National Renewable Energy Laboratory (NREL), U.S. Department of Energy (April 2014. Available to download from: http://www.nrel.gov/docs/fy14osti/59195.pdf).

[37] D. Dorrell, A. Knight, R. Betz, Improvements in brushless doubly fed reluctance generators using high-flux-density steels and selection of the correct pole numbers, IEEE Trans. on Magnetics 47 (10) (2011) 4092-4095.

[38] H. Ye, W. Pei, Z. Qi, Analytical modeling of inertial and droop responses from a wind farm for short-term frequency regulation in power systems, IEEE Transactions on Power Systems 31 (5) (2016) 3414-3423.

[39] Rate of change of frequency (RoCoF) modification to the grid code, Tech. rep., The Commission for Energy Regulation (May 2016).

[40] R. Doherty, A. Mullane, G. Nolan, D. J. Burke, A. Bryson, M. O’Malley, An assessment of the impact of wind generation on system frequency control, IEEE Transactions on Power Systems 25 (1) (2010) 452-460. 